\title{
Electron heating in subpicosecond laser interaction with overdense and near-critical plasmas
}

\author{
L. Cialfi, ${ }^{*}$ L. Fedeli, and M. Passoni \\ Department of Energy, Politecnico di Milano University, Milan, Italy
}

(Received 1 August 2016; published 4 November 2016)

\begin{abstract}
In this work we investigate electron heating induced by intense laser interaction with micrometric flat solid foils in the context of laser-driven ion acceleration. We propose a simple law to predict the electron temperature in a wider range of laser parameters with respect to commonly used existing models. An extensive two-dimensional (2D) and 3D numerical campaign shows that electron heating is due to the combined actions of $\mathbf{j} \times \mathbf{B}$ and Brunel effect. Electron temperature can be well described with a simple function of pulse intensity and angle of incidence, with parameters dependent on pulse polarization. We then combine our model for the electron temperature with an existing model for laser-ion acceleration, using recent experimental results as a benchmark. We also discuss an exploratory attempt to model electron temperature for multilayered foam-attached targets, which have been proven recently to be an attractive target concept for laser-driven ion acceleration.
\end{abstract}

DOI: 10.1103/PhysRevE.94.053201

\section{INTRODUCTION}

Laser-driven ion acceleration is an active research topic, which has attracted significant theoretical and experimental efforts in recent decades [1,2]. Research activity in this field aims at providing compact ultrashort sources of high-energy ions for a variety of potential applications such as hadrontherapy [3], neutron and radionuclides production [4-6], and proton fast ignition [7]. Moreover, besides these foreseen applications, laser-driven ion sources already find use in experiments as a tool to investigate the time-resolved structure of electromagnetic fields [8].

Different schemes for laser-driven ion acceleration have been proposed, such as radiation pressure acceleration (RPA), breakout after burner (BOA), and collisionless shock acceleration (CSA). Most of them are characterized by challenging experimental requirements: ultrathin targets and ultrahigh laser contrast for RPA, careful control of plasma density for CSA, extreme control of laser-plasma coupling for BOA [1,2]. On the other hand, the widely investigated target normal sheath acceleration (TNSA) scheme stands out for its robustness $[6,9,10]$ and for its far less demanding experimental requirements. TNSA is arguably the most natural acceleration scheme, to the point that, not infrequently, it must be suppressed in order to observe other acceleration mechanisms [11].

In TNSA, the laser-plasma interaction generates hot electrons, which expand through the target. As they cross the rear surface a strong electrostatic field $(\mathrm{TV} / \mathrm{m})$ is formed due to charge separation. This leads to the acceleration of ions from the rear surface, to energies exceeding tens of $\mathrm{MeV}$ [12]. Accelerated ions in TNSA are typically originated from hydrocarbon contaminants of the rear surface, but they can come also from the bulk of the target or from a specifically prepared coating layer [1,2].

The generation of hot electrons is of crucial importance for ion acceleration. However, this process is known to depend strongly on several experimental parameters. In experiments with ultraintense $\left(I>10^{18} \mathrm{~W} / \mathrm{cm}^{2}\right)$, ultrashort (sub

*lorenzo.cialfi@polimi.it ps) pulses and sharp-edged micrometric plain solid targets (SLTs) two collisionless mechanisms dominate the electron heating [13,14]: the Brunel effect and $\mathbf{j} \times \mathbf{B}$ heating. In both cases, electrons are extracted from the skin layer and reinjected into the target by the action of the normal component of the electric field in the former and the oscillating component of the $\mathbf{j} \times \mathbf{B}$ force in the latter. The Brunel effect should vanish completely at normal incidence and for $S$ polarization since there is no component of the electric field normal to the surface in both cases, while it is increasingly important for higher incidence angles. Instead, $\mathbf{j} \times \mathbf{B}$ heating is active at all incidence angles for $P$ and $S$ polarization, while it is strongly quenched for $C$ polarization due to the suppression of the oscillating component of the $\mathbf{j} \times \mathbf{B}$ force.

Although it is well known that many experimental parameters of both the laser and the target can deeply affect hot electron generation (and thus ion acceleration), the role played by many of them is not satisfactorily understood yet. In this framework, numerical simulations are extremely powerful tools to achieve a better understanding of the physical processes at play. They provide information on quantities (e.g., phase space and fs-nm resolved EM fields) not measurable in actual experiments. However, they can be computationally expensive [ 40k CPU hours for each three-dimensional (3D) simulation performed for this work], and special care should be taken if a quantitative agreement with experimental results is desired.

On the other hand, theoretical ion acceleration models offer quick and simple estimations of few important features (such as the maximum energy of the ions) for given laser and target parameters. These models, however, are difficult to extend to all the possible experimental configurations and at the state of the art they are suitable only for simple cases (e.g., SLT with no polarization or angle of incidence dependencies). Most theoretical models rely on simple scaling laws to estimate the so-called hot electron temperature $T_{e}$. One of the most frequently used is the ponderomotive scaling [14] $T_{e}[\mathrm{MeV}]=0.511\left(\sqrt{1+a_{0}^{2} / 2}-1\right)$. However, this approach has some clear limitations: no polarization or incidence angle dependence, which are known to be crucial for laser-plasma coupling [15]. Thus, a proper extension of this scaling law 
is needed to improve current theoretical models. This is, however, not straightforward and is especially challenging if we consider innovative target designs that have been proposed and studied in recent years [16-21]. Among them, multilayered targets (MLTs) created by the deposition of a low-density foam on the irradiated surface of a solid target have been proven to be interesting both theoretically and experimentally [22-24].

In this work we propose a simple model for the electron temperature as a function of pulse intensity, polarization, and angle of incidence of the laser. The model is supported by an extensive two-dimensional (2D) and 3D particle-in-cell (PIC) simulation campaign and its predictions, suitably combined with an existing ion acceleration model, have been benchmarked against recent experimental results [23,24]. We also discuss an exploratory investigation of the possibility of using simple scaling laws for electron temperature with multilayer targets. Also in this case, experimental results [23,24] have been used to test our model.

\section{THEORETICAL METHODS}

A large collection of theoretical models has been proposed to estimate some of the most important features of laseraccelerated ions. Three main branches of TNSA models are defined by the different treatment reserved to the ion dynamic: quasistationary [25], dynamical [26], and hybrid [27].

A model that provides a good agreement with experimental results in a wide range of conditions [28] is the quasistationary description proposed in Refs. [25,28]. This model gives an estimation of the ions cutoff energy in TNSA, which reads as follows:

$$
\begin{aligned}
E_{\max } & =Z T_{e}\left[\varphi^{*}-1+\frac{\beta\left(\varphi^{*}\right)}{I\left(\varphi^{*}\right) e^{\left(\zeta+\varphi^{*}\right)}}\right] \\
& =Z T_{e} f\left(\varphi^{*}\right)[\mathrm{MeV}],
\end{aligned}
$$

where $Z$ is the charge of the accelerated ions, $\varphi^{*}=\phi / T_{e}$ is the normalized potential deep inside the target, $\zeta=m_{e} c^{2} / T_{e}$, $\beta(x)=\sqrt{(x+\zeta)^{2}-\zeta^{2}}$, and $I(x)=\int_{0}^{\beta(x)} e^{-\sqrt{\zeta^{2}+p^{2}}} d p . \varphi^{*}$ is a semiempirical quantity, whose dependence on some experimental parameters such as target thickness and pulse energy has been investigated in Ref. [29]. In Ref. [25] the authors use the well-known ponderomotive scaling [14] to estimate $T_{e}$ in Eq. (1). Besides the strong limitations highlighted in the introduction, recent works [30-32] suggest that ponderomotive scaling can significantly overestimate $T_{e}$ at high laser intensities $\left(I>10^{19} \mathrm{~W} / \mathrm{cm}^{2}\right)$. Clearly, an improvement of the estimation of $T_{e}$ would allow, not only to extend the predicting capability of this quasistationary model, but also to improve other theoretical descriptions.

While being extremely interesting from a theoretical point of view, an analytical approach similar to that presented in Ref. [31] is not straightforward to be extended at different incidence angles or new target designs and thus we have followed a different strategy, which is discussed in detail in Sec. III A.

\section{NUMERICAL SETUP}

To support the theoretical analysis, we performed an extensive 2D and 3D numerical campaign with the open-source particle-in-cell code PICCANTE [33]. The numerical activity was carried out at the HPC BG/Q machine FERMI (CINECA, Bologna, Italy).

Due to the high computational cost of 3D PIC simulations (more than 40000 CPU hours each), we mostly relied on 2D simulations, exploiting 3D to address several specific cases. We simulated $0.5 \mu \mathrm{m}$ thick targets with a charge over mass ratio $Z / A=1 / 3$ (e.g., $\left.\mathrm{Al}^{9+}\right)$, a density of $n_{e}=80 n_{c}\left(n_{c}=\right.$ $m_{e} \omega^{2} / 4 \pi e^{2}$ is the critical density) in $2 \mathrm{D}$ and $n_{e}=40 n_{c}$ in $3 \mathrm{D}$ (we performed 2D convergence tests and we observed no substantial differences between $n_{e}=80 n_{c}$ and $n_{e}=40 n_{c}$ ). A thin $\mathrm{H}^{+}$contaminant layer $\left(l_{\text {cont }}=50 \mathrm{~nm}, n_{\text {cont }}=9 n_{c} Z / A=\right.$ 1) was present at the rear side of the target. We also performed several simulations with a $20 \mu \mathrm{m}$ thick target to assess the role played by the target thickness.

The laser pulse was Gaussian shaped in the transverse direction with $\sin ^{2}$ temporal envelope, characterized by a pulse duration (intensity FWHM) of $25 \mathrm{fs}$, a waist of $3 \mu \mathrm{m}$ and a wavelength of $0.8 \mu \mathrm{m}$. The peak intensity was varied in the range of $4.8 \times 10^{18}-4.8 \times 10^{20} \mathrm{~W} / \mathrm{cm}^{2}$, which corresponds to a normalized laser amplitude $a_{0}=\sqrt{\frac{I \lambda^{2}}{1.37 \cdot 10^{18}}} \sim 1.5-15$, well within the capabilities of present facilities [34].

The role of laser polarization has been studied considering $P$ and $C$ polarized pulses at different incidence angles $\left(0-15-30-45-60^{\circ}\right)$ and intensities with $2 \mathrm{D}$ simulations (more than $602 \mathrm{D}$ simulations were performed). We also exploited 3D simulations at specific laser configurations to check possible dimensionality related effect. $S$-polarized laser pulses can not be realistically reproduced in $2 \mathrm{D}$, since the electric field is orthogonal to the simulation plain. Therefore, for this case, we performed a few 3D simulations at a fixed laser intensity $\left(a_{0}=10\right)$ with $0-30-45^{\circ}$ incidence angle.

The spatial resolution in the $2 \mathrm{D}$ campaign was $\Delta x=c / 2 \omega_{p}$ and $\Delta y=c / 1.5 \omega_{p}$ evaluating $\omega_{p}$ for the dense foil. In $3 \mathrm{D}$ simulations we adopted a moving window configuration combined with a stretched grid in the $y-z$ axis with a constant resolution in the interaction volume of $\Delta x=\Delta y=$ $\Delta z=c / \omega_{p}$. Benchmark 2D simulations proved that a lower resolution (similar to the $3 \mathrm{D}$ case) does not strongly affect the results (less than $5 \%$ on ion maximum energy).

The solid layer was sampled with 81 macroelectrons per cell and nine macroions in 2D, while in 3D 42 macroelectrons were used. The contaminant layer contained nine macroelectrons and nine macroions per cell for both $2 \mathrm{D}$ and $3 \mathrm{D}$ simulations.

Since our work relies strongly on $T_{e}$ estimated from numerical simulations, it is worth clarifying the procedure that we have followed. As illustrated in Fig. 1, we performed a linear fit in a semilogarithmic scale. All the analyzed spectra with SLT exhibit an exponential behavior $\propto e^{-E / T}$ (except for a very small number of back-accelerated electrons [35]). More complex spectra were observed for foam-attached targets (e.g., due to highly energetic electrons forward accelerated by the laser). However, we report that electrons that effectively drive TNSA are characterized by an exponential spectrum in a large energy interval. Simulations were stopped at $170 \mathrm{fs}$ $(60 \lambda / c)$, since most of the ion acceleration process in $3 \mathrm{D}$ has 


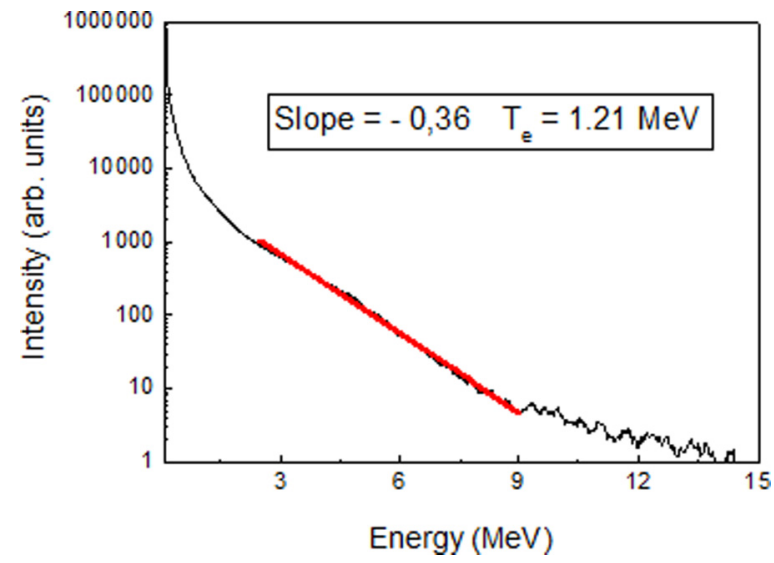

FIG. 1. An example of electron temperature estimation: in black a representative spectrum, in red its linear fit.

taken place at this point $\left(\frac{d E_{\max } \text { (ions) }}{d t \cdot E_{\max } \text { (ions) }}<1 \%\right.$ per laser period). In $2 \mathrm{D}$ simulations point charges are actually charged wires, thus their electrostatic potential is $[\propto-\ln (r)]$, which leads to a slower saturation. However, $\frac{d E_{\max } \text { (ions) }}{d t \cdot E_{\max } \text { (ions) }}$ is still less than $<5 \%$ per laser period.

\section{A. Micrometric plain foil targets}

To extend Eq. (1) to a wider range of laser parameters we propose a hot electron temperature scaling law given by two contributions associated to the $\mathbf{j} \times \mathbf{B}$ heating and Brunel effect: $T_{e}=C_{1} T_{\mathbf{j} \times \mathbf{B}}+C_{2} T_{\text {Brunel }}$.

For the $\mathbf{j} \times \mathbf{B}$ contribution we start from the well-known ponderomotive scaling:

$$
T_{\mathbf{j} \times \mathbf{B}}[\mathrm{MeV}]=0.511\left[\sqrt{1+\frac{a_{0}^{2}}{2}}-1\right] \text {. }
$$

As far as the Brunel term is of concern, no scaling laws for $T_{e}$ have been proposed. However, the simple model presented in Ref. [36] allows to estimate the interaction efficiency $\left(\eta_{b}=\right.$

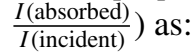

$$
\eta_{b}=\frac{1}{\pi a_{0}} f\left[\left(1+f^{2} \frac{a_{0}^{2}}{2} \sin ^{2} \theta\right)^{1 / 2}-1\right] \tan \theta,
$$

where $f$ is the field amplification factor due to the reflection of the electromagnetic wave at the solid-vacuum interface ( $f=2$ for perfect reflection on a flat surface). In general $f$ depends on the absorption efficiency $(f=1+\sqrt{1-\eta})$, however, for laser interaction with flat targets absorption efficiency is usually very low (e.g., $\eta<20 \%$ in all our simulations), thus $f$ can be safely approximated as if the pulse reflection was perfect. We can estimate the Brunel contribution as $T_{\text {Brunel }} \propto E_{\text {laser }} \eta_{b} / N_{e}$, where $N_{e}$ is the total number of electrons interacting with the laser beam, which we assume to be proportional to the relativistic skin depth $N_{e} \propto \lambda_{s d}\left(\lambda_{s d}=c / \omega_{p e} \sqrt{1+a_{0}^{2} / 2}\right)$. This leads to the following expression for the Brunel contribution:

$$
\begin{aligned}
& T_{\text {Brunel }}[\mathrm{MeV}] \\
& \propto 0.511\left[\sqrt{\left(1+f^{2} \frac{a_{0}^{2}}{2}\right)\left(\sin ^{2} \theta-1\right)}\right] \tan \theta .
\end{aligned}
$$

In Eq. (4) a factor $a_{0} / \sqrt{1+a_{0}^{2} / 2}$ should appear. However, even for modest laser intensities $\left(a_{0}>3\right)$ this term approaches a constant.

Combining Eq. (2) and Eq. (4), we propose the following scaling law for $T_{e}$, able to take into account both $\mathbf{j} \times \mathbf{B}$ and Brunel effect:

$$
\begin{aligned}
T_{e}[\mathrm{MeV}]= & C_{1}\left(a_{0}, \text { pol }, l_{\text {foil }}\right) 0.511\left[\sqrt{1+\frac{a_{0}^{2}}{2}}-1\right] \\
& +C_{2}\left(a_{0}, \text { pol }, l_{A l}\right) 0.511 \\
& \left.\times\left[\sqrt{\left(1+f^{2} \frac{a_{0}^{2}}{2}\right)\left(\sin ^{2} \theta\right.}-1\right)\right] \tan \theta,
\end{aligned}
$$

where $C_{1}\left(a_{0}, p o l, l_{\text {foil }}\right)$ and $C_{2}\left(a_{0}, p o l, l_{\text {foil }}\right)$ are the weights of the $\mathbf{j} \times \mathbf{B}$ heating and Brunel effect respectively, depending in principle on the laser polarization, intensity and target thickness. However, we assume to have included most of the dependence on the laser intensity and incidence angle explicitly in Eq. (5) and, thus, we expect $C_{1}$ and $C_{2}$ to be quasiconstant with respect to $a_{0}$.

In Fig. 2, $T_{e}$ (estimated as described in Sec. III) is shown for the three laser polarizations as a function of the incidence angle. Electron heating at normal incidence should be attributed to $\mathbf{j} \times \mathbf{B}$ heating only, while the increase of $T_{e}$ observed in Figs. 2(a) and 2(b) at higher incidence angles is mainly due to the Brunel effect. As expected, $T_{e}$ for $S$ polarization [Fig. 2(c)] does not depend on the incidence angle.

It is well known that the simulation dimensionality is likely to influence numerical results, with a 3D simulation being obviously more representative of the actual physical process. The comparison of $2 \mathrm{D}$ and $3 \mathrm{D}$ results showed that electron temperature ratio $R=T_{e}(2 D) / T_{e}(3 D)$ is weakly dependent on parameters such as intensity and incidence angle (less than $10 \%$ in our cases), but somehow sensible to the laser polarization, being $R(P$ pol $) \sim 1.5$ and $R(C p o l) \sim 1$.

It is worth to stress that, in agreement with recent works [30-32], our results confirm that the ponderomotive scaling largely overestimates the electron temperature (e.g., $\left.T_{\text {pond }}\left(a_{0}=10\right)=3.1 \mathrm{MeV}\right)$ in all our cases of study.

Electron heating might depend on target thickness, thus we performed a few tests to approach this problem. We adopted two different target configurations: $0.75 \mu \mathrm{m}$ target (thin), thick enough to suppress relativistic transparency (which can take place when the relativistic skin depth exceeds the actual thickness of the target) at all explored intensities, and a $20 \mu \mathrm{m}$ one (thick), still suitable for laser ion acceleration experiments. Similar electron spectra were found with a temperature decrease less than $20 \%$ with a 27 times thicker target.

Numerical simulations also allow to track particle trajectories, which can provide useful insights on the physical processes at play. Figures 3(a) and 3(b) show some representative electron trajectories for both targets. When the 
(a)

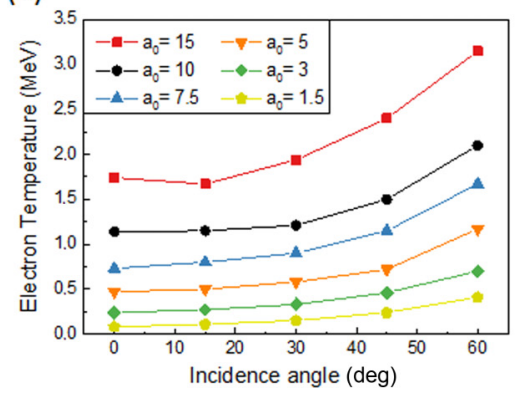

(b)

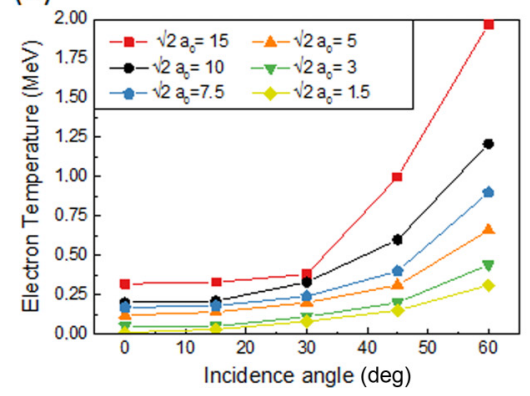

(c)

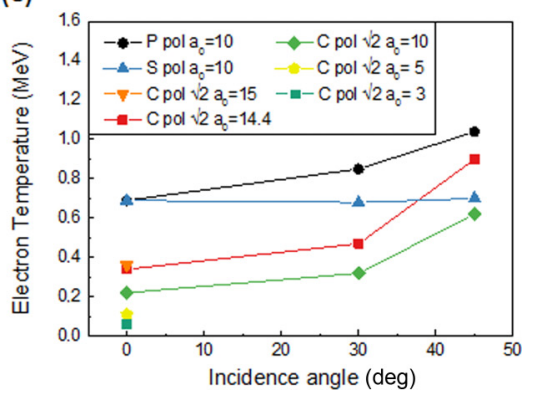

FIG. 2. Electron temperature for an irradiated a plain solid target $\left(l_{\text {foil }}=0.5 \mu \mathrm{m}, n_{e}(2 D)=80 n_{c}, n_{e}(3 D)=40 n_{c}\right)$ as function of the incidence angle at different laser intensities: a) $\mathrm{P}$ polarization b) $\mathrm{C}$ polarization c) $3 \mathrm{D}$ results.

target thickness is sufficiently small (depending on the pulse duration), electrons can recirculate multiple times in the target due to electrostatic reflection at the solid-vacuum interface. The stronger confinement of electrons in the thin target with respect to the thick one is evident, leading to a denser electron cloud and thus to a more efficient ion acceleration [29].

Electron recirculation leads to random reinjection, making trajectories hard to interpret. Thus, some features of the interaction can be seen more clearly in the thick target. Figures 3(a) and 3(b) show that electrons undergo (i) small normal oscillations near the surface, (ii) extraction in vacuum over distances of the order of the Debye length, (iii) a strong kick along the incidence angle, and (iv) injection in the target.

Figure 3(c) shows the total number of electrons along the laser propagation direction (integrated in the transverse direction) with energies above the electron temperature. The top graph shows electron bunches injected at $2 \omega$ with a spatial envelope similar to that of the pulse, which is a clear signature of $\mathbf{j} \times \mathbf{B}$ heating. In the bottom graph, electrons are injected at $2 \omega$, but it is evident that higher peaks appear with a frequency equal to $\omega$ (expected for Brunel heating). This observation supports our claim of a combined heating effect. Equation (5) captures this feature when both $C_{1}$ and $C_{2}$ are nonzero. The strategy adopted to obtain these coefficients is to exploit Eq. (5) to fit the numerical data obtained from the 2D campaign. For each simulated laser intensity, a fit of $T_{e}$ is performed, leaving $C_{1}$ and $C_{2}$ as free parameters. For each case a very good agreement with numerical results was obtained. In Figs. 4(a), 4(b) the two coefficients are shown as a function of the laser intensity. They are nearly constant for normalized laser intensities exceeding $a_{0}=3$. This confirms that our model includes most of the laser intensity dependency explicitly. At low intensity, an implicit dependence on the laser intensity in $C_{2}$ appears. In this condition $\left(I<10^{19} \mathrm{~W} / \mathrm{cm}^{2}\right)$

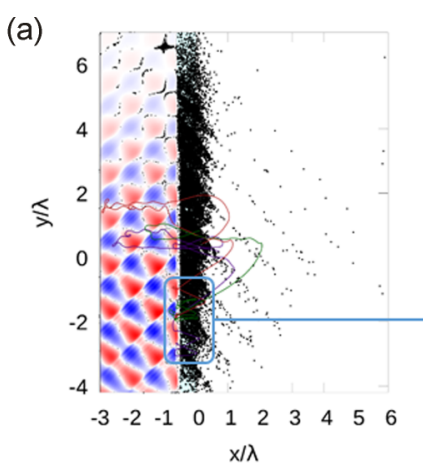

(b)

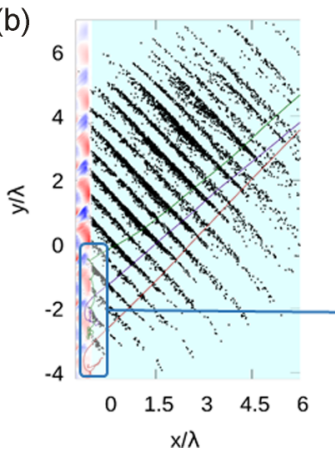

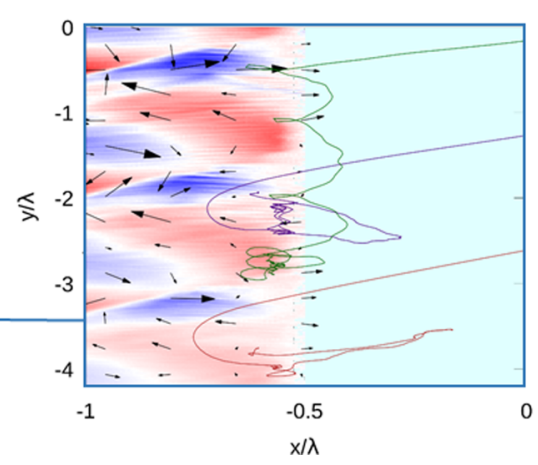

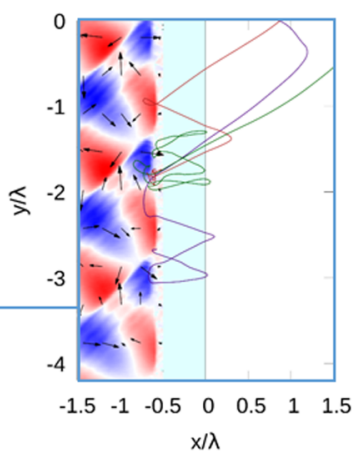

(c)

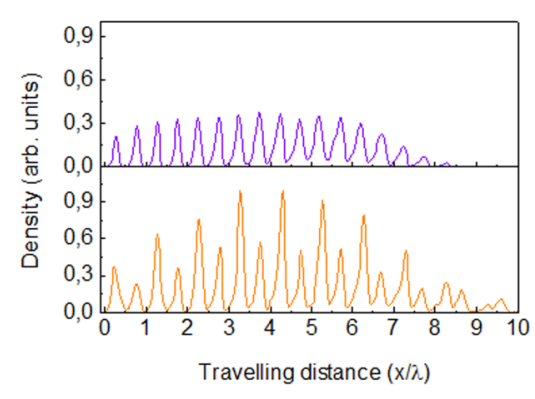

FIG. 3. Trajectories of three test electrons (colored lines) with energy above $3 \mathrm{MeV}$ after the interaction with a laser beam of $a_{0}=10$ at $45^{\circ}$ incidence with (a) $0.5 \mu \mathrm{m}$ thick target and (b) $20 \mu \mathrm{m}$. The magnetic field component $B_{z}$ (red and blue color scale) and electric field (black vectors) are taken at the injection time. The black dots represent all the electrons with an energy above $3 \mathrm{MeV}$. (c) Electron density along the laser propagation axis at normal (top) and $45^{\circ}$ incidence angle (bottom). 
(a)

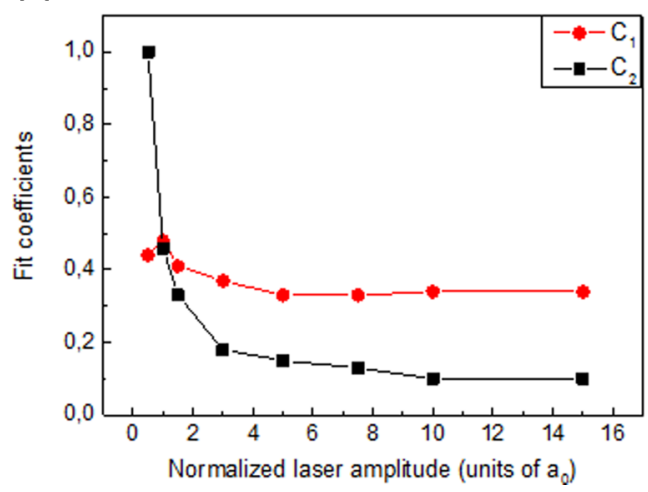

(b)

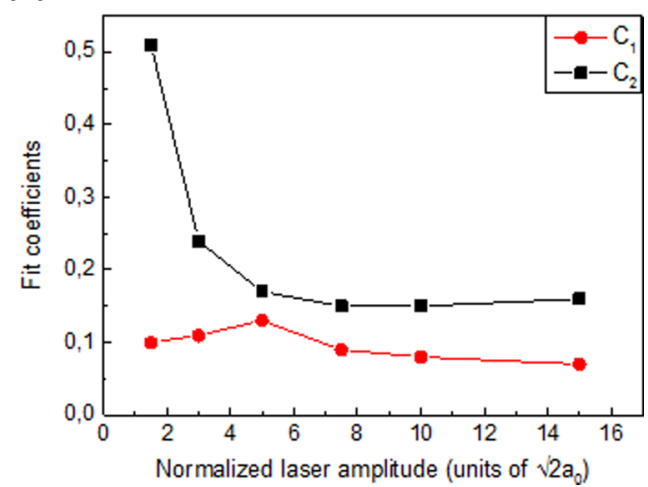

FIG. 4. $C_{1}$ (2D) [red circles] and $C_{2}$ (2D) [black squares] coefficients as function of the laser intensity for: (a) $P$ polarization, (b) $C$ polarization.

other heating mechanisms can become relevant [36], thus our combined heating model does not include all the physical processes at play. Based on these results we consider the following estimates to be reliable for $a_{0}>3$ intensities, achievable with sub $100 \mathrm{TW}$ laser facilities:

\begin{tabular}{lcc}
\hline \hline & $P$ & $C$ \\
\hline$C_{1}(2 D)$ & 0.33 & 0.1 \\
$C_{2}(2 D)$ & 0.06 & 0.16 \\
\hline \hline
\end{tabular}

3D simulations provide the best estimation for the electron temperature and are essential for $S$ polarization. However, due to the high computational cost, an extensive parametric scan could not be performed.

We selected a few representative cases for $C$ and $P$ polarization and observed that, as previously mentioned, the ratio $R[P$ pol $]=T_{e}(2 D) / T_{e}(3 D)=1.5$ and $R[C$ pol $]=1$ are weakly dependent on laser parameters. We then estimate $C_{1,2}(3 D)$ coefficients as $C_{1,2}(2 D) / R$. As far as $S$ polarization is concerned, since no dependence on the angle of incidence is observed $C_{2}=0$. At $0^{\circ}$ incidence $S$ and $P$ polarization are indistinguishable, then $C_{1}(P)=C_{1}(S)$. This procedure leads to:

\begin{tabular}{lccc}
\hline \hline & $P$ & $S$ & $C$ \\
\hline$C_{1}(3 D)$ & 0.22 & 0.22 & 0.1 \\
$C_{2}(3 D)$ & 0.04 & 0 & 0.16 \\
\hline
\end{tabular}

Equation (5) can be combined with ion acceleration models. However, since in all our cases of study $T_{e}<T_{\text {pond }}$, models that rely on the ponderomotive scaling must be changed accordingly. In the specific case reviewed in Sec. II, $\varphi^{*}$ is a semiempirical parameter, which was obtained assuming ponderomotive scaling for $T_{e}$ and fitting several experimental data. If we use Eq. (5), $\varphi^{*}$ has to be recalculated. This leaves unaltered theoretical predictions at normal incidence and $S$ polarization.

We tested Eq. (5) combined with the quasistationary model comparing its predictions with the experimental data reported in Ref. [23] (shown in Fig. 5). These results have been obtained by our group at the PW-class laser facility APRI (GIST, Gwangju, Republic of Korea) and are described in detail elsewhere $[23,24]$. In the experiment $0.75 \mu \mathrm{m}$ thick plain $\mathrm{Al}$ foils were irradiated at a fixed incidence angle $\left(30^{\circ}\right)$ using a 30 fs laser pulse with $P, C$, and $S$ polarization. As far as results for $P$ polarization are of concern [Fig. 5(a)], predictions obtained with our electron temperature model are (a)

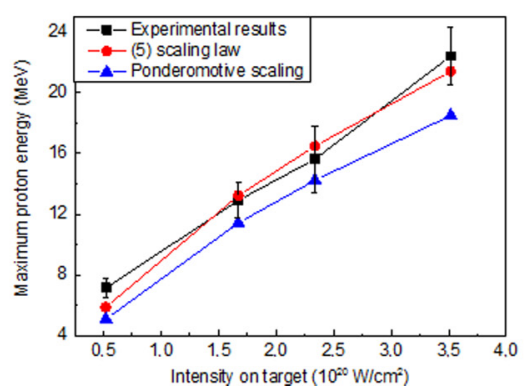

(b)

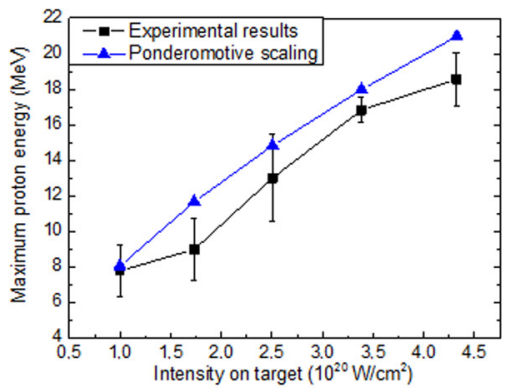

(c)

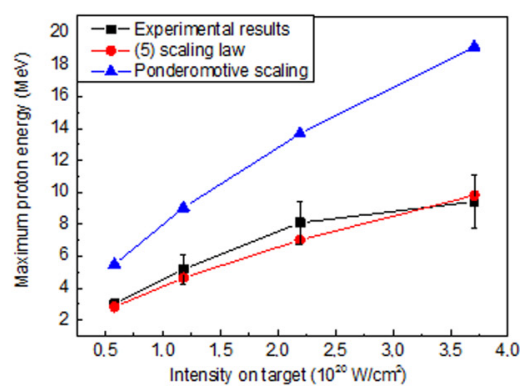

FIG. 5. Comparison between experimental results in Ref. [23] (black squares) and the quasistationary analytical model using the ponderomotive scaling (blue triangles) and the scaling 5 (red circles) for: (a) $P$ polarization, (b) $S$ polarization, and (c) $C$ polarization. 
(a)

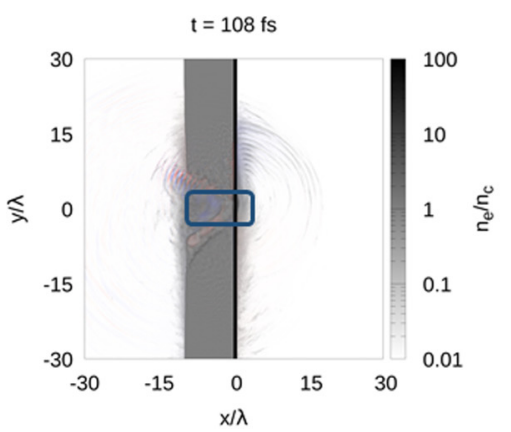

(b)

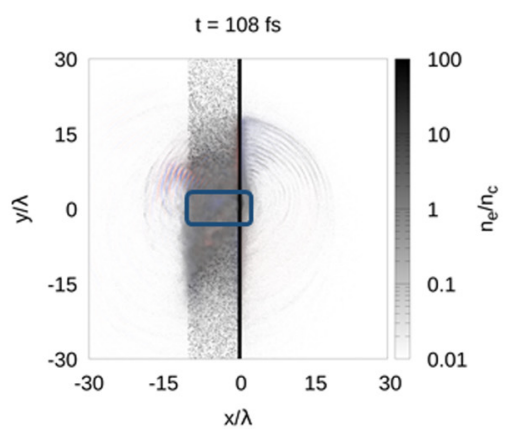

(c)

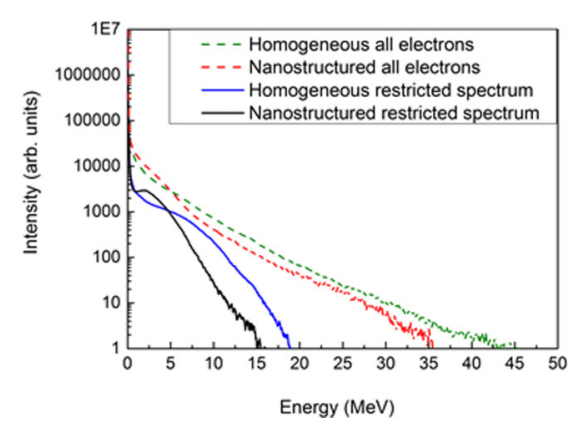

FIG. 6. Snapshots of numerical simulations for (a) homogeneous foam and (b) nanostructured foam, taken at 108 fs. The $z$ component of the magnetic field is in red and blue color scale while the electron density is in grayscale. (c) Electron spectrum at $t=108$ fs. The restricted spectrum refers to electrons taken inside the blue boxes in (a) and (b).

closer to the experimental data than the predictions relying on the ponderomotive scaling. In $S$ polarization [Fig. 5(b)] no further improvement is to be expected since $\mathbf{j} \times \mathbf{B}$ is the main heating mechanism, already described by the old scaling up to a constant factor absorbed in $\varphi^{*}$. Finally, $C$-polarization results [Fig. 5(c)] are greatly overestimated if the ponderomotive scaling is used, since $\mathbf{j} \times \mathbf{B}$ heating is almost completely suppressed. On the other hand, the improved model allows us to have a good prediction of the proton maximum energy even in this case.

\section{B. Multilayer target}

This section reports an explorative attempt to find a simple scaling law for the electron temperature even in the much more physically rich case of laser interaction with multilayered targets consisting in a near-critical foam layer coupled with a solid density foil. Ion acceleration with these targets relies on an efficient volumetric heating in the low-density layer [37-40] combined with a neat charge separation granted by the solid foil. Under suitable conditions [19,22-24], a significant enhancement of both total accelerated charge and maximum ion energy has been observed. Interaction with these

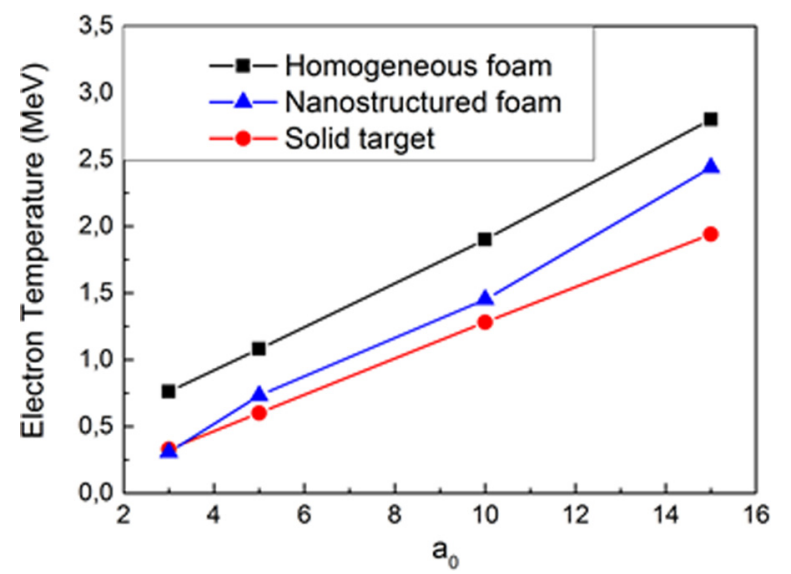

FIG. 7. Trapped electrons temperature at $30^{\circ}$ incidence for homogeneous foam (black squares), nanostructured foam (blue triangles), solid targets (red circles). complex targets is still a largely unexplored topic. Among the countless combinations of experimental parameters that can be investigated, we decided to limit this study to those of Ref. [23].

We adopted the same numerical setup presented in Sec. III, adding a $8 \mu \mathrm{m}$ thick foam in front of the thin solid target. MLT targets used in Ref. [23] were obtained growing a carbon foam on the thin solid foil with pulsed laser deposition technique [41]. These foams are characterized by a porous structure composed by solid-density nanoparticles $(d \sim 10-20 \mathrm{~nm})$ resulting in an average near-critical density on a micrometric scale. This complex structure can affect laser-plasma coupling (see Ref. [23]). As a first approach, we compared the results of numerical simulations with two different types of foam: a homogeneous plasma with $Z / A=1 / 2$ (e.g., $C^{6+}$ ) at the critical density $\left(1 n_{c}\right)$ and a nanostructured foam consisting in a spatially random collection of $10 \mathrm{~nm}$ overdense $\left(100 n_{c}\right)$ spheres, with an average density of $1 n_{c}$.

Figures 6(a), 6(b) show the electron density of the two targets taken when the total energy of the electron population reaches its peak $(t=108 \mathrm{fs})$. Laser interaction with MLT leads to a different production of hot electrons with respect to simple targets, in terms of both spectrum and spatial distribution. Two populations can be identified: electrons trapped inside the target and high-energy bunches that promptly escape. The former creates a TNSA-like electrostatic field at the solid-vacuum interface, while the latter are responsible of the quasiconstant component observed in simulations [19]. A simple 3D expanding capacitor model predicts that this component should vanish rapidly $\left(E_{x} \propto 1 /(c t)^{2}\right)$, and thus it does not contribute efficiently to the ion acceleration process. This is also confirmed by the fact that the quasiconstant field is observed only in the early stages of the interaction.

In Fig. 6(c) electron spectra of MLT targets with a homogeneous foam and a nanostructured one are reported. A clear decrease of both total number and energy is observed when only electrons trapped inside the target (and in a Debye length at the solid-vacuum interface) are considered. We assume that only these electrons can effectively drive TNSA. Figure 7 shows trapped electron temperatures with homogeneous foam, nanostructured foam and SLT. Both MLT targets exhibit an enhanced heating with respect to SLT. We remark that the nanostructure reduces the temperature with 


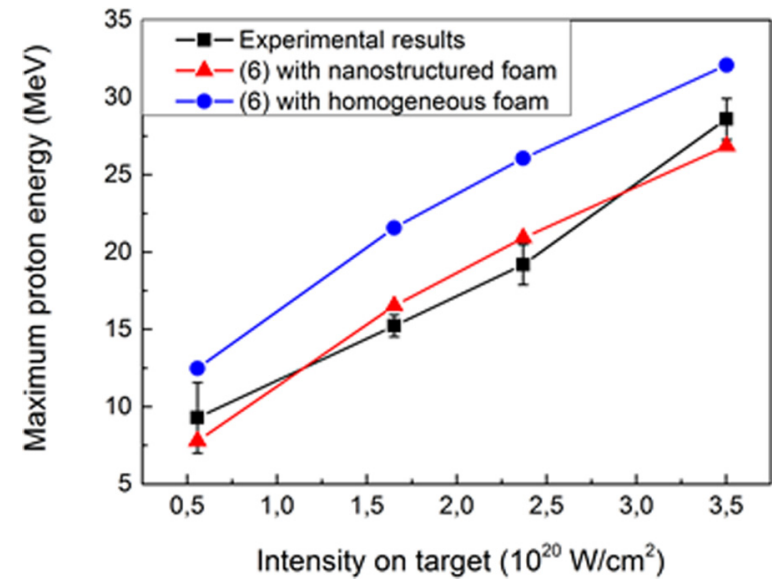

FIG. 8. Comparison between experimental results in Ref. [23] (black squares) and the analytical predictions using 6 for homogeneous foam (blue circles) and nanostructured (red triangles).

respect to the homogeneous case, which could be due to the fact that a portion of the laser energy is lost in the Coulomb explosion of the nanospheres. It is worth to highlight the linear dependence on the laser amplitude of the electron temperature even in MLT case. This linear scaling could be explained with a ponderomotivelike heating as suggested in other works involving laser interactions with near-critical plasmas [42].

In this scenario we propose a simple ponderomotivelike scaling for $T_{e}$ with two free parameters.

$$
T_{e}(M L T)=C_{3} T_{\mathrm{pond}}+C_{4} .
$$

$C_{3}$ and $C_{4}$ coefficients depend in principle on many laser and target properties. We defer a more general study to future works. Similarly to what was done in the previous section, we exploit 2D PIC simulations to fit $C_{3,4}$. Using 6 as a fit function, we obtain $C_{3}$ (homogeneous foam) $=C_{3}$ $($ nanostructured $)=0.48, C_{4}($ homogeneous foam $)=0.42$, and $C_{4}$ (nanostructured) $=0$. We then combine Eq. (6) with the quasistationary model, comparing its predictions with experimental results.

Figure 8 presents experimental results [23,24] obtained focusing a $P$-polarized laser beam at $30^{\circ}$ incidence on MLT targets $\left(l_{\text {foam }}=8 \mu \mathrm{m}, n_{\text {foam }}=1.2 n_{c}\right.$ and $\mathrm{Al} 0.75 \mu \mathrm{m}$ substrate $)$ as well as the theoretical prediction of the quasistationary model combined with Eq. (6). It worth to point out that, while using $C_{3}$ and $C_{4}$ for the homogeneous foam leads to an overestimation of the ions maximum energy, a very good agreement with experimental data is found with the coefficients referring to the nanostructured case.

This is still an early result but suggests that simple scaling laws for the electron temperature might be used even for MLT. The simulations also highlight the importance of the nanostructure in the laser-plasma coupling. Further parametric analysis will be required in order to better clarify the role of laser (incidence and polarization) and target parameters (foam density, thickness) in electron heating with complex targets, with a particular focus on more realistic nanostructured foams (e.g., using diffusion limit aggregation, as in Ref. [23]).

\section{CONCLUSIONS}

In this work we studied electron heating both with simple flat solid targets (SLTs) and multilayered foam attached targets (MLT). Combining the Brunel effect and $\mathbf{j} \times \mathbf{B}$ heating we provide a simple scaling law that overcomes some limitations of current models. Our claim of a combined heating is supported by an extensive 3D and 2D numerical campaign. Simulations suggest also that ponderomotive scaling may strongly overestimate the electron temperature.

In order to test our scaling law, we combined it with a quasistationary TNSA model. The comparison with experimental results presented in Ref. [23] shows a better agreement with respect to predictions relying on the ponderomotive scaling.

The second part of this work involved the study of electron heating with MLT, exploited for enhanced TNSA. Efficient laser-plasma coupling in the near-critical foam layer leads to higher electron temperatures with respect to SLT. A strong indication from simulations is that only electrons trapped in the target and in a Debye length at the vacuum-target interface significantly contribute to the ion acceleration. A preliminary test of different models for the near-critical layer suggest that target nanostructure can play a significant role in electron heating. However, either considering a uniform foam or a more realistic nanostructured one, a simple ponderomotivelike scaling could be found. The good agreement between model predictions and experimental results [23] is a first but promising step towards the study of laser absorption with MLT. As a final remark, target nanostructure is expected to lower laser polarization and incidence angle dependencies with respect to SLT and it is partially confirmed by recent findings [23]. This could stimulate further experimental and numerical investigations, which could lead to an improvement of our model.

In conclusion, our work gives insights on electron heating in a wide of range of experimental conditions, providing simple scaling laws for a quantity of great interest such as the electron temperature. These results are relevant for scenarios that require efficient laser-plasma coupling (e.g., laser-driven ion acceleration, studies on electron transport, etc.). Moreover, this work could stimulate experimental activities aimed at the direct measurement of the electron temperature.

\section{ACKNOWLEDGMENTS}

We want to thank Dr. Andrea Sgattoni for useful discussions and his advice. The research leading to these results has received funding from the European Research Council Consolidator Grant ENSURE (ERC-2014-CoG No. 647554). We also acknowledge ISCRA access scheme to the BlueGene/Q machine FERMI at CINECA, Italy, via the class $\mathrm{C}$ project LIonFAT.
[1] A. Macchi, M. Borghesi, and M. Passoni, Ion acceleration by superintense laser-plasma interaction, Rev. Mod. Phys. 85, 751 (2013).
[2] H. Daido, M. Nishiuchi, and A. S. Pirozhkov, Review of laserdriven ion sources and their applications, Rep. Prog. Phys. 75, 056401 (2012). 
[3] S. V. Bulanov, T. Z. Esirkepov, V. S. Khoroshkov, A. V. Kuznetsov, and F. Pegoraro, Oncological hadrontherapy with laser ion accelerators, Phys. Lett. A 299, 240 (2002).

[4] E. Amato, A. Italiano, D. Margarone, B. Pagano, S. Baldari, and G. Korn, Study of the production yields of $18 \mathrm{f}, 11 \mathrm{c}, 13 \mathrm{n}$ and 15 o positron emitters from plasma-laser proton sources at eli-beamlines for labeling of pet radiopharmaceuticals, Phys. Res. A 811, 1 (2016).

[5] D. P. Higginson, L. Vassura, M. M. Gugiu, P. Antici, M. Borghesi, S. Brauckmann, C. Diouf, A. Green, L. Palumbo, H. Petrascu et al., Temporal Narrowing of Neutrons Produced by High-Intensity Short-Pulse Lasers, Phys. Rev. Lett. 115, 054802 (2015).

[6] S. Kar, A. Green, H. Ahmed, A. Alejo, A. P. L. Robinson, M. Cerchez, R. Clarke, D. Doria, S. Dorkings, J. Fernandez, S. R. Mirfayzi, P. McKenna, K. Naughton, D. Neely, P. Norreys, C. Peth, H. Powell, J. A. Ruiz, J. Swain, O. Willi, and M. Borghesi, Beamed neutron emission driven by laser accelerated light ions, New J. Phys. 18, 053002 (2016).

[7] J. C. Fernández, B. J. Albright, F. N. Beg, M. E. Foord, B. M. Hegelich, J. J. Honrubia, M. Roth, R. B. Stephens, and L. Yin, Fast ignition with laser-driven proton and ion beams, Nucl. Fusion 54, 054006 (2014).

[8] C. K. Li, F. H. Séguin, J. A. Frenje, J. R. Rygg, R. D. Petrasso, R. P. J. Town, P. A. Amendt, S. P. Hatchett, O. L. Landen, A. J. Mackinnon et al., Measuring E and B Fields in Laser-Produced Plasmas with Monoenergetic Proton Radiography, Phys. Rev. Lett. 97, 135003 (2006)

[9] A. Favalli, N. Guler, D. Henzlova, K. Falk, S. Croft, D. C. Gautier, K. D. Ianakiev, M. Iliev, S. Palaniyappan, M. Roth et al., arXiv:1605.05702.

[10] S. Kar, H. Ahmed, R. Prasad, M. Cerchez, S. Brauckmann, B. Aurand, G. Cantono, P. Hadjisolomou, C. L. S. Lewis, A. Macchi et al., Guided post-acceleration of laser-driven ions by a miniature modular structure, Nat. Commun. 7, 10792 (2016).

[11] F. Fiuza, A. Stockem, E. Boella, R. A. Fonseca, L. O. Silva, D. Haberberger, S. Tochitsky, C. Gong, W. B. Mori, and C. Joshi, Laser-Driven Shock Acceleration of Monoenergetic ion Beams, Phys. Rev. Lett. 109, 215001 (2012).

[12] F. Wagner, O. Deppert, C. Brabetz, P. Fiala, A. Kleinschmidt, P. Poth, V. A. Schanz, A. Tebartz, B. Zielbauer, M. Roth, T. Stöhlker, and V. Bagnoud, Maximum Proton Energy Above $85 \mathrm{MeV}$ from the Relativistic Interaction of Laser Pulses with Micrometer Thick $\mathrm{ch}_{2}$ targets, Phys. Rev. Lett. 116, 205002 (2016).

[13] T. Liseykina, P. Mulser, and M. Murakami, Collisionless absorption, hot electron generation, and energy scaling in intense laser-target interaction, Phys. Plasmas 22, 033302 (2015).

[14] S. C. Wilks and W. L. Kruer, Absorption of ultrashort, ultraintense laser light by solids and overdense plasmas, IEEE J. Quantum Electron. 33, 1954 (1997).

[15] T. Ceccotti, A. Lévy, H. Popescu, F. Réau, P. D’Oliveira, P. Monot, J. P. Geindre, E. Lefebvre, and Ph. Martin, Proton Acceleration with High-Intensity Ultrahigh-Contrast Laser Pulses, Phys. Rev. Lett. 99, 185002 (2007).

[16] S. Okihara, T. Zh. Esirkepov, K. Nagai, S. Shimizu, F. Sato, M. Hashida, T. Iida, K. Nishihara, T. Norimatsu, Y. Izawa, and S. Sakabe, Ion generation in a low-density plastic foam by interaction with intense femtosecond laser pulses, Phys. Rev. E 69, 026401 (2004).
[17] T. Ceccotti, V. Floquet, A. Sgattoni, A. Bigongiari, O. Klimo, M. Raynaud, C. Riconda, A. Heron, F. Baffigi, L. Labate, L. A. Gizzi, L. Vassura, J. Fuchs, M. Passoni, M. Květon, F. Novotny, M. Possolt, J. Prokůpek, J. Proška, J. Pšikal, L. Štolcová, A. Velyhan, M. Bougeard, P. D’Oliveira, O. Tcherbakoff, F. Réau, P. Martin, and A. Macchi, Evidence of Resonant Surface-Wave Excitation in the Relativistic Regime Through Measurements of Proton Acceleration from Grating Targets, Phys. Rev. Lett. 111, 185001 (2013).

[18] D. Margarone, O. Klimo, I. J. Kim, J. Prokůpek, J. Limpouch, T. M. Jeong, T. Mocek, J. Pšikal, H. T. Kim, J. Proška, K. H Nam, L. Štolcová, I. W. Choi, S. K. Lee, J. H. Sung, T. J. Yu, and G. Korn, Laser-Driven Proton Acceleration Enhancement by Nanostructured Foils, Phys. Rev. Lett. 109, 234801 (2012).

[19] A. Sgattoni, P. Londrillo, A. Macchi, and M. Passoni, Laser ion acceleration using a solid target coupled with a low-density layer, Phys. Rev. E 85, 036405 (2012).

[20] I. J. Kim, K. H. Pae, C. M. Kim, H. T. Kim, J. H. Sung, S. K. Lee, T. J. Yu, II. W. Choi, C.-L. Lee, K. H. Nam, P. V. Nickles, T. M. Jeong, and J. Lee, Transition of Proton Energy Scaling using an Ultrathin Target Irradiated by Linearly Polarized Femtosecond Laser Pulses, Phys. Rev. Lett. 111, 165003 (2013).

[21] J. H. Bin, W. J. Ma, H. Y. Wang, M. J. V. Streeter, C. Kreuzer, D. Kiefer, M. Yeung, S. Cousens, P. S. Foster, B. Dromey, X. Q. Yan, R. Ramis, J. Meyer-ter-Vehn, M. Zepf, and J. Schreiber, Ion Acceleration Using Relativistic Pulse Shaping in Near-CriticalDensity Plasmas, Phys. Rev. Lett. 115, 064801 (2015).

[22] M. Passoni, A. Zani, A. Sgattoni, D. Dellasega, A. Macchi, I. Prencipe, V. Floquet, P. Martin, T. V. Liseykina, and T. Ceccotti, Energetic ions at moderate laser intensities using foambased multi-layered targets, Plasma Phys. Controlled Fusion 56, 045001 (2014).

[23] M. Passoni, A. Sgattoni, I. Prencipe, L. Fedeli, D. Dellasega, L. Cialfi, I. W. Choi, I. J. Kim, K. A. Janulewicz, H. W. Lee et al., Toward high-energy laser-driven ion beams: Nanostructured double-layer targets, Phys. Rev. Accel. Beams 19, 061301 (2016).

[24] I. Prencipe, A. Sgattoni, D. Dellasega, L. Fedeli, L. Cialfi, I. W. Choi, I. J. Kim, K. A. Janulewicz, K. F. Kakolee, H. W. Lee, J. H. Sung, S. K. Lee, C. H. Nam, and M. Passoni, Development of foam-based layered targets for laser-driven ion beam production, Plasma Phys. Controlled Fusion 58, 034019 (2016).

[25] M. Passoni and M. Lontano, Theory of Light-ion Acceleration Driven by a Strong Charge Separation, Phys. Rev. Lett. 101, 115001 (2008).

[26] P. Mora, Thin-foil expansion into a vacuum, Phys. Rev. E 72, 056401 (2005).

[27] B. J. Albright, L. Yin, B. M. Hegelich, K. J. Bowers, T. J. T. Kwan, and J. C. Fernández, Theory of Laser Acceleration of Light-ion Beams from Interaction of Ultrahigh-Intensity Lasers with Layered Targets, Phys. Rev. Lett. 97, 115002 (2006).

[28] M. Passoni, L. Bertagna, and A. Zani, Target normal sheath acceleration: Theory, comparison with experiments and future perspectives, New J. Phys. 12, 045012 (2010).

[29] M. Passoni, C. Perego, A. Sgattoni, and D. Batani, Advances in target normal sheath acceleration theory, Phys. Plasmas 20, 060701 (2013). 
[30] Y.-Q. Cui, W.-M. Wang, Z.-M. Sheng, Y.-T. Li, and J. Zhang, Laser absorption and hot electron temperature scalings in laserplasma interactions, Plasma Phys. Controlled Fusion 55, 085008 (2013).

[31] T. Kluge, T. Cowan, A. Debus, U. Schramm, K. Zeil, and M. Bussmann, Electron Temperature Scaling in Laser Interaction with Solids, Phys. Rev. Lett. 107, 205003 (2011).

[32] A. Flacco, F. Sylla, M. Veltcheva, M. Carrié, R. Nuter, E. Lefebvre, D. Batani, and V. Malka, Dependence on pulse duration and foil thickness in high-contrast-laser proton acceleration, Phys. Rev. E 81, 036405 (2010).

[33] A. Sgattoni, L. Fedeli, S. Sinigardi, A. Marocchino, A. Macchi, V. Weinberg, and A. Karmakar, Optimising PICCANTE - an Open Source Particle-in-Cell Code for Advanced Simulations on Tier-0 Systems, PRACE white paper 1 (2015).

[34] C. Danson, D. Hillier, N. Hopps, and D. Neely, Petawatt class lasers worldwide, High Power Laser Science and Engineering 3, e3 (2015).

[35] M. Thévenet, A. Leblanc, S. Kahaly, H. Vincenti, A. Vernier, F. Quéré, and J. Faure, Vacuum laser acceleration of relativistic electrons using plasma mirror injectors, Nat. Phys. 12, 355 (2016).

[36] P. Gibbon, Short Pulse Laser Interaction with Matter (Imperial College Press, London, 2005), p. 328.
[37] D. B. Zou, H. B. Zhuo, X. H. Yang, F. Q. Shao, Y. Y. Ma, T. P. Yu, H. C. Wu, Y. Yin, Z. Y. Ge, and X. H. Li, Enhanced target normal sheath acceleration based on the laser relativistic self-focusing, Phys. Plasmas 21, 063103 (2014).

[38] L. Willingale, P. M. Nilson, A. G. R. Thomas, S. S. Bulanov, A. Maksimchuk, W. Nazarov, T. C. Sangster, C. Stoeckl, and K. Krushelnick, High-power, kilojoule laser interactions with near-critical density plasma, Phys. Plasmas 18, 056706 (2011).

[39] D. Jung, B. J. Albright, L. Yin, D. C. Gautier, R. Shah, S. Palaniyappan, S. Letzring, B. Dromey, H.-C. Wu, T. Shimada, R. P. Johnson, M. Roth, J. C. Fernandez, D. Habs, and B. M. Hegelich, Beam profiles of proton and carbon ions in the relativistic transparency regime, New J. Phys. 15, 123035 (2013).

[40] S. S. Bulanov, V. Yu. Bychenkov, V. Chvykov, G. Kalinchenko, D. W. Litzenberg, T. Matsuoka, A. G. R. Thomas, L. Willingale, V. Yanovsky, K. Krushelnick, and A. Maksimchuk, Generation of gev protons from 1 pw laser interaction with near critical density targets, Phys. Plasmas 17, 043105 (2010).

[41] A. Zani, D. Dellasega, V. Russo, and M. Passoni, Ultra-low density carbon foams produced by pulsed laser deposition, Carbon 56, 358 (2013).

[42] A. P. L. Robinson, R. M. G. M. Trines, J. Polz, and M. Kaluza, Absorption of circularly polarized laser pulses in near-critical plasmas, Plasma Phys. Controlled Fusion 53, 065019 (2011). 